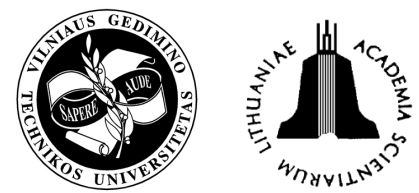

\title{
VALUING ATTRIBUTES OF ENHANCED TRAFFIC INFORMATION: AN EXPERIENCE IN KOLKATA
}

\author{
Debasis Basu ${ }^{1}$ and Bhargab Maitra ${ }^{2}$ \\ Dept of Civil Engineering, Indian Institute of Technology, Kharagpur 721 302, India \\ E-mails: ${ }^{1}$ basudebasis2k@gmail.com; ${ }^{2}$ bhargab@civil.iitkgp.ernet.in
}

Received 26 January 2007; accepted 2 May 2007

\begin{abstract}
Most of the traffic information considers a single item like travel time or delay. In the present work, enhanced traffic information displaying instantaneous travel time and its variation from the previous interval to the present, is considered. An initial investigation is made on the effectiveness of such traffic information on route choice behavior of trip makers by valuation of attributes of the traffic information. Taking a case study of two urban corridors in the Kolkata metro city, India, the valuation is done separately for private car and taxi trip makers. The stated preference (choice based) data collected from trip makers are analyzed using both multinomial logit (MNL) and mixed logit (ML) modeling techniques. Assuming sparsely used constrained triangular distribution of random parameters, two different types of ML model are developed: one with independent choice sets and the other one by accounting heterogeneity around the mean of random parameter(s). Both family income and trip purpose are found to decompose heterogeneity around the mean estimate(s). The values of travel time and their variation presented in the paper encourage further investigation on such type of traffic information for management of congestion on alternative urban corridors both spatially and temporally.
\end{abstract}

Keywords: instantaneous traffic information, value of travel time and travel time variation, stated choice, multinomial logit, mixed logit, constrained T-distribution, preference heterogeneity.

\section{Introduction}

In recent years, there has been an increasing trend of providing traffic information to trip makers using road-side Variable Message Sign (VMS) board for spatial and temporal management of road traffic congestion [15]. Accordingly, research on the applicability and suitability of VMS system has also grown in many facets. One of the facets of research for a successful application of VMS is on the content and its format to be displayed $[6,7]$. Though there have been a lot of works on the content of information and its format, yet it is found from the established literature that most of the works have focused on content including a single item like travel time or delay. The travel time information displayed on VMS board could be instantaneous travel time or predictive travel time. Functional performance of traffic information displaying instantaneous travel time depends on the possible fluctuation in demand curve if other related factors [8] remain unchanged throughout the analysis period. In reality, there may be unequal fluctuations in instantaneous travel time on alternative routes over a time period due to not only the approach traffic volume at the decision point (where the traffic information is displayed) but also other roadway and traffic factors (e. g. route length, number of intersections, entry and exit traffic volumes at intersections, capacities of different road links and intersections forming a route etc.). Such situations are often observed on alternative urban corridors in developing countries. In such situations, providing enhanced traffic information containing more than one item might be helpful for trip makers to make rational route choice decision.

The objective of the present work is to suggest enhanced traffic information containing more than one item, and make an initial investigation on the effect of such traffic information on the route choice behavior of trip makers. The effect of enhanced traffic information on route choice behavior will primarily depend on the valuing of displayed travel attributes by trip makers. Therefore, an initial investigation on the effect of such traffic information system on the route choice behavior of trip makers is attempted through the valuation of travel attributes displayed as traffic information. The valuation of travel attributes is attempted using stated preference data collected from the trip makers. Travel time is the single largest factor used as traffic information for spatial and temporal management of traffic congestion on alternative urban corridors. Therefore, instantaneous travel time is considered in the present 
work as one item for display in the VMS board. The change in instantaneous travel time from the previous interval to the present is considered as another item for display in the VMS board. It is thought that if enhanced traffic information includes not only the instantaneous travel time but also its variation from the previous interval to the present, then trip makers would be able to make more rational route choice decision.

For valuing of instantaneous travel time and its variation, two competitive traffic corridors in the Kolkata Metro City, India are considered as a case study where the traffic information is considered to be displayed on a road side VMS board placed at the junction of two corridors. No other traffic information systems are considered to exist along these corridors to act as en-route traffic information provider. For the purpose of valuing, a stated choice (SC) method is adopted to elicit preferences by generating hypothetical profiles using various levels of instantaneous travel time, levels of variation of that travel time and travel cost attributes. Instantaneous travel time is presented in quantitative format (in minute) and its variation is presented in qualitative format (such as Very High/High/Moderate/Low). During the stated choice experimentation, respondents are also informed about the quantitative basis used for defining the levels of variation. In the course of developing the utility model, both the Multinomial Logit (MNL) and Random Parameter Logit (RPL) techniques are explored. The study also takes a measure to observe the presence of any heterogeneity effect on the coefficients estimate and its subsequent effect on the valuation.

The paper is organized as follows. The methodology section introduces the approach and theoretical basis which is followed by empirical analysis. Next, the results of the analysis and conclusion are presented respectively.

\section{Methodology}

\subsection{Approach}

In discrete choice models valuing of an attribute measure is relatively straightforward as given by the ratio of partial derivatives of the utility function with respect to that attribute and travel cost (i.e. marginal rate of substitution between the attribute and travel cost at constant utility). The linear utility functions based on fixed taste coefficients are used for estimating value of time (VOT) and value of its variation (VOV). Indeed, the deterministic part $V$, of the utility function in the model contains travel-time attribute TT, its variation attribute VT and travel-cost attribute TC. Then VOT and VOV are simply computed as follows:

$$
\begin{aligned}
& \mathrm{VOT}=\frac{\partial V / \partial \mathrm{TT}}{\partial V / \partial \mathrm{TC}} ; \\
& \mathrm{VOV}=\frac{\partial V / \partial \mathrm{VT}}{\partial V / \partial \mathrm{TC}}
\end{aligned}
$$

with the commonly used linear-in-variables of the utility function, the above formulas reduce to $\beta_{T T} / \beta_{T C}$ and $\beta_{V T} / \beta_{T C}$ respectively, where $\beta_{T T}, \beta_{V T}$ and $\beta_{T C}$ are the coefficients of travel time information, its variation and travel cost. It is important to appreciate that the justification for this approach rests on a substantial body of microeconomic theory that addresses the issue of how individuals allocate time and its variation amongst alternatives.

In order to develop the utility model it is necessary to collect preferences of trip makers either in the form of Revealed Preference (RP) or Stated Preference (SP) data. RP and/or SP data have been used in diverse fields for valuing of attributes [9-15]. With a richer disaggregation of travel time, RP data is usually inappropriate. It is best described as "dirty" from the point of view of statistical estimation of the individual preferences on choice. Some attribute levels may not be observed in RP data and the predictor variables (attributes of alternatives, contextual effects) may exhibit high or extreme levels of multicollinearity consequent on market forces, technology and sampling considerations [16]. On the other hand, SP data are rich and effective for valuing purpose [17]. In SP experiment, a systematic combination of levels of each attribute can be done to reveal new opportunities (i.e. new travel scenarios) relative to the existing circumstances of time-cost on offer [16]. Therefore, in the present work, SP data is used for the development of utility model. However, SP experiments have many features that can influence the resulting value of time and its variations. In particular it is thought that the estimates are sensitive to the design of the SP experiment [16], especially, (a) the number of alternatives in a choice set, (b) the number of choice sets (treatments) evaluated and (c) the range and levels of attributes being traded. Though SP data may be collected in the form of rating, ranking and choice, stated choice (SC) experiments provide a framework for studying the relative marginal disutility of variations in attributes [18].

Generally, SP and/or RP, data are analyzed using traditional Multinomial Logit (MNL) models due to simplicity in estimation. However, the MNL model imposes some restrictions such as independence of irrelevant alternatives (IIA). It is seen that valuing of attributes may likely be under-estimated in MNL model because an element of the unobserved influences on travel choices is "forced" into the parameter estimates of the observed effects when the strict independently and identically distributed (IID) condition of standard MNL model is imposed on the utility function. Theory suggests that this has impact on the time attributes more than the cost attribute because many of the unobserved attributes are more correlated with travel time than with travel cost $[19,20]$. From the perspective of econometrics, it can be said that the mean of a random parameter is likely to be larger than that of MNL because the random parameter logit model decomposes the unobserved component of utility [16]. Therefore, modifications to the MNL model 
to reduce the influence of these restrictions lead to Random Parameter Logit (RPL)/Mixed Logit (ML)/Random Coefficient Logit (RCL) models.

\subsection{Theoretical basis and econometric models}

In econometric models based on random utility theory [21, 22], the utility of each element consists of an observed (deterministic) component denoted by $V$ and a random (disturbance) component denoted by $\varepsilon$,

$$
U=V+\varepsilon \text {. }
$$

The deterministic part $V$ is again a function of the observed attributes $z$ of the choice as faced by the individual, the observed socioeconomic attributes of the individual $S$ and a vector of coefficients $\beta$, then:

$$
V=V(z, S, \beta) \text {. }
$$

A probabilistic statement can be made (due to presence of the random component) as, when an individual " $n$ " is facing a choice set, $C_{n}$, consisting of $J_{n}$ choices, the choice probability of alternative $\mathrm{i}$ is equal to the probability that the utility of alternative " $i$ ", $U_{i n}$, is greater than or equal to the utilities of all other alternatives in the choice set, i.e.:

$$
\begin{aligned}
P_{n}(i)= & \operatorname{Pr}\left(U_{i n} \geq U_{j n}, \text { for all } j \in C_{n}\right) ; \\
P_{n}(i)= & \operatorname{Pr}\left(V_{i n}+\varepsilon_{i n} \geq V_{j n}+\varepsilon_{j n},\right. \\
& \text { for all } \left.j \in C_{n}, \forall j \neq i\right) .
\end{aligned}
$$

Assuming IID (Gumbel distribution) for $\varepsilon$, the probability that an individual chooses $i$ can be given by the Multinomial Logit Model (MNL) [22, 23],

$$
P_{i n}=\frac{e^{V_{i n}}}{\sum_{j \in C_{n}} e^{V_{i n}}} .
$$

This model can be estimated by Maximum Likelihood technique, and is useful for modeling choice behavior. However, several limitations apply to this model. The most severe of these, is the IIA property, which states that a change in the attributes of one alternative changes the probabilities of the other alternatives in proportion. This substitution pattern may not be realistic in all settings. Secondly, the coefficients of all attributes are assumed to be the same for all respondents in a choice experiment, whereas in reality there may be substantial variability in how different individuals respond to attributes. Actually there is a need to introduce a conventional form of heterogeneity of preferences to understand the interactions between alternative attributes and individual socioeconomic characteristics such as gender, age, income level, trip purpose [24-26]. In this case the parameter of each attribute is required to be a function of the individuals' observed socioeconomic characteristics as mentioned, which allows us to detect systematic variations in tastes. However, on many occasions individual information is not available, or tastes may vary with characteristics that are difficult to measure or can- not be observed. In these cases, (4) can be generalized to consider heterogeneity specifying random parameters for each individual. Thus, the utility of alternative $i$ for an individual $n$ would be:

$$
u_{i n}=\beta_{n} x_{i n}+\varepsilon_{i n}=\bar{\beta} x_{i n}+\hat{\beta}_{n} x_{i n}+\varepsilon_{i n} .
$$

Thus, each individual's coefficient vector $\beta_{n}$ is the sum of the population mean $\bar{\beta}$ and individual deviation $\hat{\beta}_{n}$ from the average value for the population. $\hat{\beta}_{n} x_{i n}$ is the error component that induces heteroskedasticity in the unobserved portion of the utility. This implies an important implication of Random Parameter Logit (RPL) specification, where we do not have to assume the IIA property holds. In the above equation (8) $x_{i n}$ are observed variables that relate to the alternative and individual, and $\beta_{n}$ is a vector of coefficients of these variables for individual $n$. Let the coefficients vary over individuals in the population with density $f\left(\beta_{n} \mid \theta\right)$, where $\theta$ represents the mean and covariance of $\beta_{n}$ in the population and if the error term $\varepsilon_{i n}$ follows the IID type I extreme value distribution, then the RPL model is called Mixed Logit (ML) Random Parameter Model [27] because then the choice probability is the mixture of logits with $f$ as mixing distribution [28].

In this case, the individual knows the value of her/his own $\beta_{n}$ and $\varepsilon_{i n}$ 's for all $i$ and chooses alternative $i$ if and only if $U_{i n} \geq U_{j n}$ for all $J \in C_{n}, \forall j \neq i$. On the other hand the modeler/researcher observes $x_{i n}$ 's but not $\beta_{n}$ or the $\varepsilon_{i n}$ 's. If the modeler observed $\beta_{n}$, then the choice probability would be standard logit, since the $\varepsilon_{i n}$ 's are IID type I extreme value distribution. Then the probability, conditional on $\beta_{n}$ is:

$$
L_{i n}\left(\beta_{n}\right)=\frac{\exp \left(\beta_{n} x_{i n}\right)}{\sum_{i=1}^{J} \exp \left(\beta_{n} x_{i n}\right)} .
$$

However, the modeler does not know $\beta_{n}$, and therefore the probability cannot be conditional. Therefore the unconditional choice probability is the integral of $L_{\text {in }}\left(\beta_{n}\right)$ over all possible variables of $\beta_{n}$ [27].

$$
P_{i n}=\int\left(\frac{\exp \left(\beta_{n} x_{i n}\right)}{\sum_{i=1}^{J} \exp \left(\beta_{n} x_{i n}\right)}\right) f\left(\beta_{n} \mid \theta\right) d \beta_{n} .
$$

The ML model does not have a closed form expression (unlike the MNL model) and so it is approximated numerically through simulation by the method of Simulated Maximum Likelihood (SML). Here a simulated maximum likelihood estimator, using Halton draws, is used to estimate the models [28-30]. This procedure offers the potential to reduce the number of 
draws that are needed for estimation of ML models, thereby reducing run times, and/or reducing simulation error that is associated with a given number of draws $[16,29,30]$. This type of random parameter model is less restrictive than standard conditional logit model. However, these less restrictive models should be applied cautiously. Apart from being more difficult to estimate, literature shows that the results can be rather sensitive to the distributional assumptions and the number of draws applied in the simulation [31].

\subsection{Selection of distribution}

In RPL/ML model, it is necessary to make an assumption regarding the distribution of each of the random coefficients. The choice of distribution is often limited by difficulty of model estimation and availability of econometric software. The alternative distributions available are normal, log-normal, uniform and triangular. The lognormal form is often used if the mean of random parameter needs to be a specific (non-negative) sign. A disadvantage of lognormal form is that it has a long upper tail. A uniform distribution with a $(0,1)$ bound is sensible when dummy variables are to be estimated. For the triangular distribution, the density function looks like a tent: a peak in the centre and dropping off linearly on both sides of the center. The disadvantage with normal, uniform and triangular distributions is that they give the wrong signs to some shares due to spread or standard deviation of the distributions. This can be avoided by imposing a constraint on the distributions so that the mean is equal to the spread [31]. In the present work a constrained triangular distribution, which is a generalization of the Uniform distribution, is instrumented for estimation of attributes. A constrained triangular distribution has a peak in the density function with two endpoints of the distribution fixed at zero and two times at the mean, so that there is no free variance (scaling) parameter. Although the constrained triangular distribution has several advantages over the other distributions, its application in valuing has not been explored adequately. In the present work, the application of constrained triangular distribution is explored with ML models.

\section{Empirical study}

\subsection{Study area}

Two distinct and competitive traffic corridors are selected in the Kolkata Metro City. For the study corridors, origin and destination are selected based on catchment area concept, rather than zoning concept. The origin of both traffic corridors is at Park Circus and the destination is at Esplanade/Dalhousie area. One traffic corridor (called as Fly-Over corridor, FO) starts at Park Circus and then goes via the newly constructed fly-over to Rabindra Sadan and then via Red Road to Esplanade/Dalhousie area. This corridor is almost a free flow corridor and the length is $5 \mathrm{~km}$. The alternative traffic corridor (called as Park Street Corridor, PS) goes via Park Street, and the length of this corridor is $3.4 \mathrm{~km}$. The PS corridor becomes highly congested during the peak hours. From the reconnaissance survey, it is observed that both the traffic corridors carry only private cars and taxis. Also, it is found that a large amount of traffic enters at the Park Circus junction in the morning peak hours from different locations of the Kolkata city to go to Esplanade/Dalhousie area. It is required to mention that there are few entry and exit points on both of these corridors. But because of the city's traffic network and the existing traffic guidelines (one-way traffic system), those points are not the escape points for Esplanade/Dalhousie bound traffic. So, there exists no as such possibility for Esplanade/Dalhousie bound traffic to get off the corridor en route.

\subsection{Survey instrument}

Survey instruments were designed to collect the respondents' socio-economic data, present corridor choice behavior, trip characteristics and the stated choice responses. The three attributes considered for the SP study are travel time, its variation level and travel cost. Depending on the possible variation in instantaneous travel time from one time interval to the next, four levels of variation are defined as follows:

- Low: If the change in instantaneous travel time from the previous time interval to the present is within $20 \%$ of the instantaneous travel time.

- Medium: If the change in instantaneous travel time from the previous time interval to the present is within $20-30 \%$ of the instantaneous travel time.

- High: If the change in instantaneous travel time from the previous time interval to the present is within $30-40 \%$ of the instantaneous travel time.

- Very high: If the change in instantaneous travel time from the previous time interval to the present is within $40-50 \%$ of the instantaneous travel time.

Based on reconnaissance survey and discussions with regional traffic experts, traffic police, taxi drivers and trip makers, the levels of the attributes (Table 1 and Table 2) for the two corridors, are selected.

The levels of cost attribute for the private car trip makers in the SP experiment are designed based on diesel and gasoline fuel types, present fuel cost and possible price hike in the future. Although the design of experiment is done taking all the levels of cost attribute irrespective of fuel type, but during the survey, private car trip makers were interviewed with the appropriate questionnaire set considering the type of fuel they use. It is observed that among all the private car trip makers, almost $80 \%$ use gasoline. Therefore, a common model is developed for all private car trip makers irrespective of the fuel types they use. It is a common practice to de- 
Table 1. Attributes and their levels for private car trip makers

\begin{tabular}{|c|l|c|}
\hline Corridor & \multicolumn{1}{|c|}{ Attribute } & Levels \\
\hline \multirow{3}{*}{ Fly-Over } & $\begin{array}{l}\text { Travel time } \\
\text { (min) }\end{array}$ & $5,10,15$ \\
\cline { 2 - 3 } & Level of variation & Low, Medium \\
\cline { 2 - 3 } & Taxi Fare (Rs.) & $15,25,35,45,55$ \\
\hline \multirow{3}{*}{ Park Street } & $\begin{array}{l}\text { Travel time } \\
\text { (min) }\end{array}$ & $10,15,20,25,30,35,40$ \\
\cline { 2 - 3 } & Level of variation & Medium, High, Very High \\
\cline { 2 - 3 } & Taxi fare (Rs.) & $5,15,25,35,45$ \\
\hline$* 1$ USD 45 INR (Indian Rupee) \\
\hline
\end{tabular}

Table 2. Attributes and their levels for taxi trip makers

\begin{tabular}{|c|l|c|}
\hline Corridor & \multicolumn{1}{|c|}{ Attribute } & Levels \\
\hline \multirow{4}{*}{ Fly-Over } & Travel time (min) & $5,10,15$ \\
\cline { 2 - 3 } & Level of variation & Low, Medium \\
\cline { 2 - 3 } & Taxi fare (Rs.)* & $40,45,50,55,60,65$ \\
\hline \multirow{3}{*}{ Park Street } & Travel time (min) & $10,15,20,25,30,35,40$ \\
\cline { 2 - 3 } & Level of variation & Medium, High, Very High \\
\cline { 2 - 3 } & Taxi fare (Rs.)* & $30,35,40,45,50,55$ \\
\hline *1 USD $~ 45$ INR (Indian Rupee) \\
\hline
\end{tabular}

velop alternatives using either full factorial or fractional factorial design. However, it was neither necessary nor practically possible to include all the combinations resulting from full factorial design in the SP experiment. Therefore, some of the choice sets were eliminated using fractional factorial orthogonal technique without loss of much of statistical properties of full factorial design. Fractional factorial/orthogonal main effects only design $[18,32]$ reduced the number of combinations effectively by eliminating some of the higher order combinations. Fractional factorial orthogonal design using SPSS 7.5 [28] was used to produce the alternatives. A sum of 80 alternatives was generated for each of two corridors for different trip makers. Subject to preservation of orthogonality, the levels of attributes for both SP alternatives (corridors) were "swapped" to ensure that neither Fly-Over corridor nor Park Street corridor dominates each other [16]. This way 80 competitive choice sets were generated for each of the two types of trip makers. To reduce the confusion and/or fatigue of respondents, these 80 choice sets were grouped randomly into 16 blocks, each containing 5 choice sets (observations). This way 16 (i.e. $80 / 5=16$ ) different sets of questionnaire were developed. The questionnaire consisted of two parts. The first part was common to all 16 questionnaires and to collect respondents' socioeconomic status, present travel behavior and trip characteristics whereas, the second part was to collect the stated choice responses.

\subsection{Database development}

A choice based stated preference survey was carried out in the Kolkata City in the month of May-June,
2005. A paper-pencil based survey was carried out as face-to-face interviews with private car and taxi trip makers. Before carrying out the survey, a team of ten enumerators was selected and trained in multiple sessions. Their understanding about the questionnaire and the ways of explaining the questionnaire to trip makers were checked by conducting several pilot surveys. The main survey and data collection proceeded only after satisfactory performance of enumerators during the final round of the pilot survey.

During the survey, trip makers were intercepted at various predetermined strategic locations in the city. Enumerators explained to each respondent about the study as well as the attributes and their levels used in the choice experiment. Respondents willing to participate in the survey were then interviewed and requested to provide their socioeconomic data and the choice of the corridor taken for the most-recent trip between Park Circus and Esplanade/Dalhousie area in the light of perceived travel time and travel cost on alternative corridors.

Finally, the choice response was also taken for each of the five choice sets/ observations given in the questionnaire.

During the data collection from taxi trip makers, only the post-paid taxi trip makers were interviewed as for such taxi trips the route choice decision is generally made by trip makers themselves. More than $90 \%$ of taxis operating in Kolkata are metered taxis (i.e. postpaid) and therefore, it was possible to intercept adequate number of post-paid taxi trip makers. During the data collection, 1100 Private Car trip makers and 1500 taxi trip makers were interviewed.

However, for consistency in the responses and completeness, the data collected from 955 private car and 1074 taxi trip makers were used for the model development purpose. Some descriptive statistics about the database are presented in Table 3.

\subsection{Model development}

For the development of the utility models, 4643 and 5035 observations were used for private car and taxi trip makers respectively. In the present paper, utility models are developed using both instantaneous travel time and its variation in quantitative format (i.e. in minutes). The travel time variation is expressed in minutes by taking the mid-value of the defined variation level and multiplying it by the corresponding instantaneous travel time. Alternative-specific intercept is included for the Fly-Over corridor. For analyzing behavioral data, the less restrictive Mixed Logit (ML) technique is employed along with the Multinomial Logit (MNL) technique using LIMDEP 8.0 [33]. Two different sets of ML models are estimated; one with independent choice sets, and the other by assuming heterogeneity (i.e. effect of socioeconomic variables such as income, trip purpose etc.) around the mean of the random parameters. Private car and taxi trip makers are split into two groups based on the monthly family income. Private car users with monthly family income of 
Table 3. Descriptive statistics

\begin{tabular}{|c|c|c|c|c|}
\hline Socioeconomic variables & \multicolumn{2}{|c|}{ Private car } & \multicolumn{2}{|c|}{ Taxi } \\
\hline \multirow{2}{*}{ Gender } & Male & $87 \%$ & Male & $69 \%$ \\
\hline & Female & $13 \%$ & Female & $31 \%$ \\
\hline \multirow{4}{*}{ Age } & $<20$ years & $2 \%$ & $<20$ years & $29 \%$ \\
\hline & 20 to 35 years & $40 \%$ & 20 to 35 years & $52 \%$ \\
\hline & 36 to 55 years & $52 \%$ & 36 to 55 years & $16 \%$ \\
\hline & $>55$ years & $6 \%$ & $>55$ years & $3 \%$ \\
\hline \multirow{3}{*}{ Education } & Upto $10^{\text {th }}$ standard & $10 \%$ & Upto $10^{\text {th }}$ standard & $4 \%$ \\
\hline & Upto $12^{\text {th }}$ standard & $26 \%$ & Upto $12^{\text {th }}$ standard & $33 \%$ \\
\hline & Graduate/Master level & $64 \%$ & Graduate/Master level & $63 \%$ \\
\hline \multirow{2}{*}{ Monthly family income } & $<=$ Rs. $20,000 /-$ & $64 \%$ & $<=$ Rs. $10,000 /-$ & $31 \%$ \\
\hline & > Rs. $20,000 /-$ & $36 \%$ & $>$ Rs. $10,000 /-$ & $69 \%$ \\
\hline \multirow{3}{*}{ Household size } & $<3$ & $13 \%$ & $<3$ & $17 \%$ \\
\hline & 3 to 5 & $74 \%$ & 3 to 5 & $75 \%$ \\
\hline & $>5$ & $13 \%$ & $>5$ & $8 \%$ \\
\hline \multirow{2}{*}{ Trip purpose } & Revenue generating & $29 \%$ & Revenue generating & $38 \%$ \\
\hline & Non-revenue generating & $71 \%$ & Non-revenue generating & $62 \%$ \\
\hline
\end{tabular}

up to Rs.20,000/- (1USD 45 INR) are considered as low income, and beyond Rs.20,000/- are considered as high income. For taxi users, monthly family income of up to 10,000/- and beyond Rs.10,000/- are used to define low income and high income respectively. Private car and taxi trip makers are also split into two groups based on trip purpose. Trips are classified as revenue generating trips (i.e. work and business trips) and nonrevenue generating trips (i.e. social, recreational and other trips).

In all the ML models, the cost coefficient is considered as non-random because of the following reasons: (a) it simplifies the estimation of value of time and its variation, i.e. valuing is simple division of the coefficient of an attribute by the coefficient of cost, (b) the distribution of the marginal value for travel time/its variation is simply the distribution of that attribute, and (c) the travel cost attribute is restricted to be nonpositive for all individuals. The alternative specific intercept is also kept as non-random. On the other hand, the coefficients of travel time and its variation are assumed to be randomly distributed following the constrained T-distribution [34]. Here a simulated maximum likelihood estimator, using Halton draws, is used to estimate the model $[28,29]$.

\subsection{Results and discussion}

Coefficient estimates from the MNL and the ML techniques for private car trip makers are given in Table 4, while Table 5 shows the same for taxi trip makers. In ML models with preference heterogeneity, family income per month and trip purpose, are found to decompose heterogeneity around the mean of the random parameter(s). It is observed from Table 4 and Table 5 that the signs of the parameter estimates are as expected and are in agreement with the actual scenario of the study corridors. The coefficient estimates of all attributes are negative representing all the attributes as disutility. It is evident from the t-statistics of parameters that they are statistically (more than $95 \%$ or $90 \%$ confidence level) significantly different from zero. The overall goodness of fit is considered using pseudo $R^{2}$ $\left(\rho^{2}\right)$. The $\rho^{2}$ values of all models indicate that these models are a good fit. However, no significant change in $\rho^{2}$ is observed between MNL and ML model specifications. In fact the $\rho^{2}$ is observed to vary in a close range of 0.2404 to 0.2508 for models of private car trip makers. For models of taxi trip makers, the $\rho^{2}$ is found to vary in the range of 0.1989 to 0.2301 . Therefore, both MNL and ML specifications in the present work are found to indicate nearly the same model fit to the observed data.

The interpretation of the model coefficients in Table 4 and Table 5 are not straightforward except for the significance and goodness-of-fit. So, the marginal rates of substitution between attributes and cost are calculated. Table 6 and Table 7 show the value of travel time and its variation for private car and taxi trip makers respectively. It is observed from Table 6 that when the MNL technique is employed, the values of travel time and its variation are nearly same for private car trip makers. However, when the ML technique is used, the value of travel time variation is found to be higher than that of travel time. For taxi trip makers, both MNL and ML techniques produced distinctly higher values of travel time variation than the travel time itself. It is also observed from Table 6 and Table 7 that values of instantaneous travel time from different logit models for private car trip makers are significantly higher than those of taxi trip makers. Similar observation has been reported for the value of travel time in Mumbai Metro City, India [35]. It is also noticed that values of travel time variation for taxi trip makers are significantly higher than the corresponding values for private car trip 
Table 4. Coefficients estimate from MNL and ML techniques for private car trip makers

\begin{tabular}{|c|c|c|c|c|}
\hline \multirow[b]{2}{*}{ Attribute } & \multirow{2}{*}{$\begin{array}{c}\text { MNL Model } \\
\text { Coefficient } \\
(t \text {-stat })\end{array}$} & \multicolumn{3}{|c|}{ ML Models } \\
\hline & & $\begin{array}{c}\text { Coefficient } \\
(t \text {-stat })\end{array}$ & $\begin{array}{c}\text { Coefficient } \\
(t \text {-stat })\end{array}$ & $\begin{array}{c}\text { Coefficient } \\
(t \text {-stat })\end{array}$ \\
\hline & & \multicolumn{3}{|c|}{ Random parameters (constrained T-dist.) } \\
\hline Travel time (TT) & $\begin{array}{c}-0.1493 \\
(-16.301)\end{array}$ & $\begin{array}{l}-0.20415 \\
(-11.916)\end{array}$ & $\begin{array}{l}-0.20336 \\
(-10.081)\end{array}$ & $\begin{array}{l}-0.20057 \\
(-10.046)\end{array}$ \\
\hline \multirow[t]{2}{*}{ Variation in travel time (VT) } & $\begin{array}{c}-0.14711 \\
(-6.926)\end{array}$ & $\begin{array}{l}-0.2276 \\
(-6.926)\end{array}$ & $\begin{array}{l}-0.2127 \\
(-5.622)\end{array}$ & $\begin{array}{c}-0.20663 \\
(-5.596)\end{array}$ \\
\hline & & \multicolumn{3}{|c|}{ Non random parameters } \\
\hline Travel cost (TC) & $\begin{array}{l}-0.15294 \\
(-20.706)\end{array}$ & $\begin{array}{l}-0.18378 \\
(-20.706)\end{array}$ & $\begin{array}{c}-0.18365 \\
(15.321) \\
\end{array}$ & $\begin{array}{l}-0.1841 \\
(-15.44)\end{array}$ \\
\hline \multirow[t]{2}{*}{ Asc. to $\mathrm{FO}^{\dagger}$} & $\begin{array}{c}0.553595 \\
(4.913) \\
\end{array}$ & $\begin{array}{c}0.272753 \\
(4.913) \\
\end{array}$ & $\begin{array}{c}0.285655 \\
(1.92)^{* *}\end{array}$ & $\begin{array}{c}0.24271 \\
(1.63)^{*}\end{array}$ \\
\hline & & \multicolumn{3}{|c|}{ Std div. or spread of random parameters } \\
\hline Travel time (TT) & & $\begin{array}{l}0.20415 \\
(11.916)\end{array}$ & $\begin{array}{l}0.20336 \\
(10.081)\end{array}$ & $\begin{array}{l}0.20057 \\
(10.046)\end{array}$ \\
\hline \multirow[t]{3}{*}{ Variation in travel time (VT) } & & $\begin{array}{l}0.2276 \\
(6.926)\end{array}$ & $\begin{array}{l}0.2127 \\
(5.622)\end{array}$ & $\begin{array}{c}0.20663 \\
(5.596)\end{array}$ \\
\hline & & & \multicolumn{2}{|c|}{ Heterogeneity in the mean of random parameter $(s)$} \\
\hline & & & Income & Trip purpose \\
\hline Travel time (TT) & & & - & - \\
\hline Variation in travel time (VT) & & & $\begin{array}{l}-0.0350 \\
(-2.528)\end{array}$ & $\begin{array}{c}-0.110688 \\
(-7.042)\end{array}$ \\
\hline No of observation & 4643 & 4643 & 4643 & 4643 \\
\hline Log likelihood function & -1938.97875 & -1958.995 & -1955.181 & -1931.989 \\
\hline$\rho^{2}$ & 0.24813 & 0.24036 & 0.24184 & 0.25084 \\
\hline
\end{tabular}

makers. Generally, private car trip makers are habitual users of private cars. On the contrary, trip makers generally hire taxis in the time of urgency or in an instant-need and therefore, taxi trip makers are found to value the travel time variation more than the private car trip makers. Of course, ML technique indicates generally higher order value of travel time variation over travel time for both private car and taxi trip makers. It may be mentioned that Liu et al. [36] reported a study on the contribution of travel time reliability to travelers' route choice decision. It was observed that the estimated median value of travel-time reliability was substantially greater than that of travel time, and the median value of degree of risk aversion was significantly greater than unity, indicating that travelers valued more highly a reduction in variability than in the travel time saving. The present work does not consider the travel time reliability and therefore, the results cannot be compared directly with the observations made by Liu et al. [36]. However, the higher values of the variation of travel time information, as observed in the present work, is consistent with what was reported by Liu et al. [36].
Tables 6 and 7 show that, value of travel time and its variation emanating from different ML models are higher than those from corresponding MNL models. Higher valuing of attributes from RPL models have also been reported by Train [37] for recreational demand, Alpizar and Carlsson [38] in mode choice modeling, Hensher [16] in value of travel time savings, Amador et al. [39] in willingness-to-pay for travel time savings. Generally, trip maker's choice decision is influenced by other unobserved factors (like traffic congestion level, possibility of longer delay, etc.) which are not present in the SP questionnaire as attributes, and the effects of these factors are normally more correlated with travel time and its variation rather than travel cost attribute [19, 20]. As RPL models decompose such unobserved components of utility, the mean estimates of random parameters are often higher [16]. Accordingly, the values estimated for random parameters are also higher in RPL models.

\section{Conclusions}

A new type of traffic information displaying instantaneous travel time and its variation from the previous time interval to the present is considered. An ini- 
Table 5. Coefficients estimate from MNL and ML techniques for taxi trip makers

\begin{tabular}{|c|c|c|c|c|}
\hline \multirow[b]{2}{*}{ Attribute } & \multirow{2}{*}{$\begin{array}{c}\text { MNL Model } \\
\text { Coefficient } \\
(t \text {-stat })\end{array}$} & \multicolumn{3}{|c|}{ ML Models } \\
\hline & & $\begin{array}{c}\text { Coefficient } \\
(t \text {-stat })\end{array}$ & $\begin{array}{c}\text { Coefficient } \\
(t \text {-stat })\end{array}$ & $\begin{array}{c}\text { Coefficient } \\
(t \text {-stat })\end{array}$ \\
\hline \multicolumn{5}{|c|}{ Random parameters (constrained T-dist.) } \\
\hline Travel time $(\mathrm{TT})$ & $\begin{array}{l}-0.0688 \\
(-5.698)\end{array}$ & $\begin{array}{c}-0.11319 \\
(-6.582)\end{array}$ & $\begin{array}{l}-0.1378 \\
(-4.416)\end{array}$ & $\begin{array}{c}-0.0928 \\
(-4.24)\end{array}$ \\
\hline Variation in travel time (VT) & $\begin{array}{c}-0.31863 \\
(-8.486)\end{array}$ & $\begin{array}{c}-0.41009 \\
(-5.237)\end{array}$ & $\begin{array}{l}-0.2301 \\
(-3.359)\end{array}$ & $\begin{array}{c}-0.45465 \\
(-3.587)\end{array}$ \\
\hline \multicolumn{5}{|c|}{ Non random parameters } \\
\hline Travel cost (TC) & $\begin{array}{c}-0.1489 \\
(-11.969) \\
\end{array}$ & $\begin{array}{c}-0.1797 \\
(-11.341) \\
\end{array}$ & $\begin{array}{c}-0.1814 \\
(-10.648) \\
\end{array}$ & $\begin{array}{c}-0.18339 \\
(-9.201) \\
\end{array}$ \\
\hline Asc. to $\mathrm{FO}^{\dagger}$ & $\begin{array}{l}0.7332 \\
(3.692) \\
\end{array}$ & $\begin{array}{c}0.51517 \\
(1.966) \\
\end{array}$ & $\begin{array}{c}0.4372 \\
(1.847)^{*}\end{array}$ & $\begin{array}{l}0.54725 \\
(1.823)^{*}\end{array}$ \\
\hline \multicolumn{5}{|c|}{ Std Div. or Spread of random parameters } \\
\hline Travel time (TT) & & $\begin{array}{c}-0.11319 \\
(-6.582)\end{array}$ & $\begin{array}{l}0.1378 \\
(4.416)\end{array}$ & $\begin{array}{c}0.0928 \\
(4.24)\end{array}$ \\
\hline Variation in travel time (VT) & & $\begin{array}{c}-0.41009 \\
(-5.237) \\
\end{array}$ & $\begin{array}{l}0.2301 \\
(3.359) \\
\end{array}$ & $\begin{array}{c}0.45465 \\
(3.587) \\
\end{array}$ \\
\hline \multicolumn{5}{|c|}{ Heterogeneity in the mean of random parameters } \\
\hline & & & Income & Trip purpose \\
\hline Travel time $(\mathrm{TT})$ & & & $\begin{array}{l}0.0647 \\
(2.632)\end{array}$ & $\begin{array}{c}-0.06392 \\
(-2.111)\end{array}$ \\
\hline Variation in travel time (VT) & & & $\begin{array}{l}-0.3285 \\
(-5.688) \\
\end{array}$ & $\begin{array}{l}0.117087 \\
(1.695)^{* *} \\
\end{array}$ \\
\hline No of observation & 5035 & 5035 & 5035 & 5035 \\
\hline Log likelihood function & -1444.568 & -1452.348 & -1395.711 & -1448.930 \\
\hline$\rho^{2}$ & 0.20318 & 0.19889 & 0.23013 & 0.20077 \\
\hline $\begin{array}{l}\dagger \text { Stands for fly-over corridor; } \\
* \text { Significant almost at } 95 \% \text { confi } \\
* * \text { Significant at } 90 \% \text { confidence }\end{array}$ & & & & \\
\hline
\end{tabular}

Table 6. Value of travel time and its variation for private car trip makers

\begin{tabular}{|l|c|c|c|c|c|c|}
\hline \multirow{2}{*}{ Attribute } & MNL model & ML model & \multicolumn{4}{|c|}{ ML models with heterogeneity } \\
\cline { 4 - 7 } & & & Low income & High income & $\begin{array}{c}\text { Non revenue gen- } \\
\text { erating }\end{array}$ & $\begin{array}{c}\text { Revenue gen- } \\
\text { erating }\end{array}$ \\
\hline Travel time (TT) (Rs./min.) & 0.98 & 1.11 & 1.11 & 1.11 & 1.09 & 1.09 \\
\hline $\begin{array}{l}\text { Variation in travel time (VT) } \\
\text { (Rs./min.) }\end{array}$ & 0.96 & 1.24 & 1.16 & 1.35 & 1.12 & 1.72 \\
\hline 1 USD 45 Indian Rupee (INR) and 1 INR=100 Paise
\end{tabular}

Table 7. Value of travel time and its variation for taxi trip makers

\begin{tabular}{|l|c|c|c|c|c|c|}
\hline \multirow{2}{*}{ Attribute } & \multirow{2}{*}{ MNL model } & ML model & \multicolumn{4}{|c|}{ ML models with heterogeneity } \\
\cline { 4 - 7 } & & & Low income & High income & $\begin{array}{c}\text { Non revenue gen- } \\
\text { erating }\end{array}$ & $\begin{array}{c}\text { Revenue gen- } \\
\text { erating }\end{array}$ \\
\hline Travel time (TT) (Rs./min.) & 0.46 & 0.63 & 0.76 & 0.40 & 0.51 & 0.85 \\
\hline $\begin{array}{l}\text { Variation in travel time (VT) } \\
\text { (Rs./min.) }\end{array}$ & 2.14 & 2.28 & 1.27 & 3.07 & 2.48 & 1.84 \\
\hline 1 USD 45 Indian Rupee (INR) and $1 \mathrm{INR}=100$ Paise \\
\hline
\end{tabular}

tial investigation is made into the effect of such traffic information on the route choice behaviour of trip makers through the valuation of travel time and its variation. Taking a case study of two urban corridors in
Kolkata Metro City, India, the valuation is done separately for private car and taxi trip makers. For valuation purpose, the stated choice data collected from trip makers are analyzed using different logit model specifica- 
tions. For both private car and taxi trip makers, the estimated values of travel time variation are generally found to be higher than the corresponding values of travel time implying that the travel time variation, if also projected as traffic information, would influence the route choice behaviour of both private car and taxi trip makers. The values of travel time and their variation presented in the paper encourage further investigation on such type of traffic information for management of congestion on alternative urban corridors both spatially and temporally.

In the process of valuation, the stated choice data are analyzed using both Multinomial Logit (MNL) and Mixed Logit (ML) modeling techniques. The values of travel time and their variation emanating from ML models are found to be higher than those from MNL models. From an analytical point of view, coefficient estimation technique in ML modeling is superior to that of MNL modeling as the former imposes less restriction on the error term. ML modeling technique can also account for the preference heterogeneity in the valuing of attributes. In the present study, both family income and trip purpose are found to decompose the preference/choice heterogeneity around the mean estimate of travel time and/or its variation. However, no consistent improvement in terms of fitness of the model on observed data (i.e. $\rho^{2}$ ) could be observed in the present case for ML models over MNL models.

In the development of ML models, it is necessary to assume a suitable distribution for random parameters. In the present work, ML models are developed assuming the sparsely used constrained triangular distribution for random parameters. Its application is expected to encourage researchers as an alternative to other commonly used distribution in developing ML models.

\section{References}

1. DURAND-RAUCHER, Y.; YIM, Y.; YGNACE, J. Traffic information and driver behaviour in Paris region. In Proc., Pacific Rim Transtech Conference, Seatle, ASCE, 1993, Vol 1, p. 167-169.

2. BENSON, B. G. Motorists attitudes about content of variable-message signs. Transportation Research Record, 1550, Transportation Research Board, Washington, D. C., 1996, p. 48-57.

3. RAMSAY, E. D.; CATCHPOLE, J. E.; LUK, J. Y. K. Evaluation of VicRoads' drive time system. Res. Rep. ARR 305, ARRB Transport Research, Vermont South, Victory, Australia, 1997

4. WARDMAN, M.; BONSALL, P. W.; SHIRES, J. D. Driver response to variable message signs: a stated preference investigation. Transportation Research Part C, 1997, Vol 5, No 6, p. 389-405.

5. BAOFENG, S.; ZHICAI, J.; LELEUR, S.; WENJING, Wu. Research reviews of socio-economic impact evaluation for VMS project on freeway. In Proc., 8th International IEEE Conference on Intelligent Transportation Systems, Vienna, Austria, Spe 13-16, 2005, p. 1017-1021.
6. CATCHPOLE, J., HANCOCK, A., CAIRNEY, P. Driver comprehension of formats for presenting traffic information on dynamic signs. Res. Rep. ARR 269, ARRB Transport Research, Vermont South, Victory, Australia, 1995.

7. LAI, K. H.; WONG, W. G. SP Approach toward driver comprehension of message formats on VMS. Journal of Transportation Engineering, ASCE, May-June, 2000, p. 221-227.

8. LOMAX, T.; SCHRANK, D.; TURNER, S.; MARGIOTTA, R. Selecting travel reliability measures. Texas Transportation Institute and Cambridge Systematics, Inc, 2003.

9. ADAMOWICZ, W. L.; LOUVIERE, J.; WILLIAMS, M. Combining stated and revealed preference methods for valuing environmental amenities. Journal of Environment Economics and Management, 1994, Vol 26, p. 271-292.

10. BATES, J. Stated preference technique for the analysis of transportation behavior. In Proc., World Conference of Transportation Research, Hamburg, W. Germany, 1982, p. 252-265.

11. KROES, E. P.; SHELDON, R. J. Stated preference methods: an introduction. Journal of Transport Economics and Policy, 1988, Vol 22, p. 11-25.

12. LOUVIERE, J. J. Conjoint analysis modeling of stated preferences: a review of theory, methods, recent developments and external validity. Journal of Transport Economics and Policy, 1988, Vol 22(1), p. 93-119.

13. HENSHER, D. A. Stated preference analysis of travel choices: the state of practice. Transportation, 1994, Vol 21(2), p. 107-133.

14. Jose HOLGUIN-VERAS, P. E. Revealed preference analysis of commercial vehicle choice process. Journal of Transportation Engineering, ASCE, 2002, Vol 128 (4), p. 236346.

15. PHANIKUMAR, C. V.; BASU, D.; MAITRA, B. Modeling generalized cost of travel for rural bus users: a case study. Journal of Public Transportation, 2004, Vol 7, No 2, p. 59-72.

16. HENSHER, D. A. Measurement of valuation of travel time savings. Journal of Transport Economics and Policy, Jan. 2001, Vol 35, Part I, p. 71-98.

17. HENSHER, D. A.; SULLIVAN, C. Willingness to pay for road curviness and road type. Transportation Research Part D, 2003, Vol 8, p. 139-155.

18. LOUVIERE, J. J.; HENSHER, D. A.; SWAIT, J. D. Stated choice methods. Analysis and applications. Cambridge University Press, 2000.

19. JARA-DIAZ, S. R. Time and income in travel choice: towards a microeconomic activity based theoretical framework. In Theoretical Foundations of Travel Choice Modeling. T. Garling, T. Laitila and K. Westin (eds.), Pergamon Press, Oxford, Chapter 3, 1998, p. 51-74.

20. JARA-DIAZ, S. R. Allocation and valuation of travel time savings. In D. A. Hensher and K. Button (eds.). Transport Modeling, Handbooks in Transport, Pergamon Press, Oxford, Chapter 192000.

21. THURSTONE, L. L. A law of comparative judgment. Psychological Review, 1927, 4, p. 273-286.

22. MCFADDEN, D. Conditional logit analysis of qualitative choice behavior. In P. Zarembka (ed.). Frontiers in Econometrics, New York: Academic Press, 1974, p. 105-142. 
23. BEN-AKIVA, M.; LERMAN, S. R. Discrete choice analysis: theory and applications to travel demand. MIT Press, Cambridge, 1985.

24. POLLAK, R. A.; WALES, T. J. Demand specification and estimation. Oxford University Press, New York, USA, 1992.

25. REVELT, D.; TRAIN, K. Mixed logit with repeated choices: households' choices of appliance ffficiency level. Review of Economics and Statistics, 1998, 80, p. 647-657.

26. ORTUZAR, J. de D.; WILLUMSEN, L. G. Modelling transport. $3^{\text {rd }}$ Edition, John Wiley and Sons, Chichester, 2001.

27. TRAIN, K. Discrete choice methods with simulation. Cambridge University Press, Cambridge, 2003.

28. HENSHER, D. A.; ROSE, J. M.; GREENE, W. H. Applied choice analysis: a primer. Cambridge University Press, New York, 2005.

29. TRAIN, K. Halton sequences for mixed logits. Working Paper, Department of Economics, University of California at Berkeley, 1999.

30. BHAT, C. R. Quasi-random maximum simulated likelihood estimation of the mixed multinomial logit model. Transportation Research Part B 35, 2001, p. 677-693.

31. HENSHER, D. A.; GREENE, W. H. The mixed logit model: the state of practice and warnings for the unwary. Working Paper, School of Business, University of Sidney, 2001.

32. GREEN, P. E.; KRIEGER, A. M.; WIND, Y. J. Thirty years of conjoint analysis: reflections and prospects. INTERFACES 31:3, Part 2 of 2, May-June, 2001, p. S56-S73.

33. LIMDEP 8.0. User's guide for LIMDEP 8.0, Econometrics Software, Inc., 2005.

34. PHANIKUMAR, C. V.; MAITRA, B. Willingness-to-pay and preference heterogeneity for rural bus attributes. Journal of Transportation Engineering, 2007, Vol 133, No 1, ASCE, p. 62-69.

35. Mumbai metro planning group mumbai metro study: travel demand forecasting model. Working Paper No 4, Mumbai, 1997.

36. LIU, H. X.; RECKER, W.; CHEN, A. Uncovering the contribution of travel time reliability to dynamic route choice using real-time loop data. Transportation Research Part A 38, 2004, p. 435-453.

37. TRAIN, K. Recreation demand models with taste differences over People. Land Economics, 1998, Vol 74, No 2, p. 230-239.

38. ALPIZAR, F.; CARLSSON, F. Policy implications and analysis of the determinants of travel mode choice: an application of choice experiments to metropolitan Costa Rica. Working Paper in Economics 56, Department of Economics, Göteborg University, 2001.

39. AMADOR F. J.; GONZÁLEZ R. M.; ORTÚZAR J. de D. Preference heterogeneity and willingness-to-pay for travel time savings. Transportation, 2005, 36, 6, p. 627-647. 